\title{
Peer
}

\section{Chronological age, biological age, and individual variation in the stress response in the European starling: a follow-up study}

\author{
Annie Gott ${ }^{1, *}$, Clare Andrews ${ }^{1, *}$, Maria Larriva Hormigos ${ }^{2}$, \\ Karen Spencer ${ }^{2}$, Melissa Bateson ${ }^{1}$ and Daniel Nettle ${ }^{1}$ \\ ${ }^{1}$ Centre for Behaviour and Evolution \& Institute of Neuroscience, Newcastle University, \\ Newcastle upon Tyne, UK \\ ${ }^{2}$ School of Psychology and Neuroscience, University of St. Andrews, St. Andrews, UK \\ $*$ These authors contributed equally to this work.
}

\section{ABSTRACT}

The strength of the avian stress response declines with age. A recently published study of European starlings (Sturnus vulgaris) found that a marker of biological age predicted the strength of the stress response even in individuals of the same chronological age. Specifically, birds that had experienced greater developmental telomere attrition (DTA) showed a lower peak corticosterone (CORT) response to an acute stressor, and more rapid recovery of CORT levels towards baseline. Here, we performed a follow-up study using the same capture-handling-restraint stressor in a separate cohort of starlings that had been subjected to a developmental manipulation of food availability and begging effort. We measured the CORT response at two different age points (4 and 18 months). Our data suggest a decline in the strength of the CORT response with chronological age: peak CORT was lower at the second age point, and there was relatively more reduction in CORT between 15 and $30 \mathrm{~min}$. Individual consistency between the two age points was low, but there were modest familial effects on baseline and peak CORT. The manipulation of begging effort affected the stress response (specifically, the reduction in CORT between 15 and $30 \mathrm{~min}$ ) in an age-dependent manner. However, we did not replicate

Submitted 24 July 2018 Accepted 27 September 2018 Published 23 October 2018

Corresponding author Daniel Nettle,

daniel.nettle@ncl.ac.uk

Academic editor

George Bentley

Additional Information and Declarations can be found on page 16

DOI 10.7717/peerj.5842

(c) Copyright

2018 Gott et al.

Distributed under

Creative Commons CC-BY 4.0

OPEN ACCESS the associations with DTA observed in the earlier study. We meta-analysed the data from the present and the earlier study combined, and found some support for the conclusions of the earlier paper.

Subjects Animal Behaviour, Zoology

Keywords Biological age, Stress response, Corticosterone, Developmental programming, Starlings, Telomeres

\section{INTRODUCTION}

The hypothalamic-pituitary-adrenal (HPA) axis is a highly conserved, integrated system in vertebrates that functions to prioritise immediate survival over non-essential activities in the face of acute threats. In birds, the strength of the HPA response generally declines with chronological age (Heidinger, Nisbet \& Ketterson, 2006; Heidinger et al., 2010; Wilcoxen et al., 2011; Elliott et al., 2014; Lendvai, Giraudeau \& Bo, 2015; López-Jiménez et al., 2017), possibly reflecting adaptive shifts in behavioural allocation as expected 
future lifespan reduces. However, individuals do not all age at the same rate: an individual's biological age can be either older or younger than their chronological age (Belsky et al., 2015). Biological age is by definition a better predictor of future lifespan than chronological age is. Hence, we should expect markers of individual biological age to explain variation in the strength of the stress response that cannot be explained by chronological age alone. A possible reason that early-life conditions have often been observed to influence the functioning of the adult stress response may be that early-life conditions can speed up or slow down the biological ageing process, and consequently advance or retard age-related shifts in the functioning of the stress response system.

One potential marker of biological age is the attrition of telomeres, the DNA caps at the ends of linear chromosomes (Bize et al., 2009; Bauer et al., 2018). Short telomere length has been shown to predict shorter subsequent lifespan in a range of avian (Bize et al., 2009; Heidinger et al., 2012; Barrett et al., 2013; Salmón et al., 2017; Wilbourn et al., 2018) as well as non-avian (Boonekamp et al., 2013; Wilbourn et al., 2018) species. The rate of telomere attrition is much higher during the developmental period than in adulthood, and has been shown to be accelerated by early-life adversity (Boonekamp et al., 2014; Nettle et al., 2015, 2017). Thus, the extent of telomere shortening can potentially be used as a proxy for individual differences in biological age.

In a recent study of two cohorts of adult European starlings (Sturnus vulgaris, $n=20$ and $n=31$ ), Andrews et al. (2017) showed that developmental telomere attrition (DTA; the extent of shortening of erythrocyte telomeres over the course of development) explained variation in individuals' stress responses. Specifically, individuals that were biologically older by this measure (all individuals were approximately the same chronological age) showed a lower peak level of corticosterone (CORT) in response to an acute capture-handling-restraint stressor, and also showed stronger recovery of CORT levels towards baseline between 15 and $30 \mathrm{~min}$ after the onset of the stressor. DTA was unrelated to baseline CORT. The birds studied by Andrews et al. (2017) had been subjected to experimental manipulations of early-life conditions: a manipulation of brood size for one cohort (Nettle et al., 2013), and of the focal individual's size relative to its competitors in the other cohort (Nettle et al., 2015). These manipulations affected DTA, with the more adverse treatment (having more or larger competitors respectively for the two cohorts) leading to greater telomere shortening in early life. However, it was DTA, rather than the early-life treatments themselves, that significantly predicted stress response. This suggests that DTA captures both the adversity due to the experimental manipulation, and other sources of adversity, and also incorporates the fact that individuals are differentially affected by the same external conditions. Thus, DTA is a better marker of biological ageing than any of the individual environmental or genetic factors that may influence it. The cohorts of birds were made up of quartets of siblings, which allowed the researchers to examine potential familial effects. They found evidence consistent with modest familial effects on baseline and peak CORT, but not on the change in CORT between 15 and $30 \mathrm{~min}$ after the onset of the stressor.

In light of recent focus on reproducibility in bioscience (Fidler et al., 2017), the results of Andrews et al. (2017) require replication. Moreover, the CORT response was only 
measured at one age point (around one year of age on average). Thus, it is not clear whether associations between DTA and stress response appear before, or persist after, this stage of life. Longitudinal studies in birds have found varying results with respect to whether individuals are consistent in CORT measures from one year to the next (Cockrem \& Silverin, 2002; Angelier et al., 2010; Ouyang, Hau \& Bonier, 2011; Rensel \& Schoech, 2011; Baugh et al., 2014; Lendvai, Giraudeau \& Bo, 2015). Where individual consistency is found, it is generally in the CORT response to an acute stressor rather than baseline CORT (Cockrem \& Silverin, 2002; Rensel \& Schoech, 2011). Thus, it is important to establish whether there is individual consistency over time in the CORT parameters studied in Andrews et al. (2017), as well as whether the associations of these parameters with DTA are robust.

Here, we report a follow-up study conducted with a different cohort of birds. The quartets of siblings in the current cohort were hand-reared according to a two-by-two factorial design that varied early food amount and begging effort. The rationale for this design was to attempt to isolate the independent effects of these two factors, both of which vary with brood size in nature. Previous studies of early-life adversity in altricial birds have found that developing in a large brood has long-term phenotypic consequences (Nettle et al., 2013; Boonekamp et al., 2014), but have not been able to establish exactly why. We have reported the effects of the developmental treatments on DTA in these birds elsewhere (Nettle et al., 2017). Briefly, a restricted food amount, and a higher begging effort, both significantly accelerated DTA in these birds, and did so additively. Thus, the two developmental treatments can each be seen as accelerating biological ageing, as proxied by DTA.

In the present experiments, we measured the CORT response to an acute capture-restraint-handling stressor using the same protocol as Andrews et al. (2017), but did so twice, when the birds were juveniles of approximately 4 months of age (age point 1), and adults of approximately18 months of age (age point 2). Our main aim was to replicate the key findings of Andrews et al. (2017), namely that DTA is associated with peak CORT and change in CORT between 15 and $30 \mathrm{~min}$, but not baseline CORT; that DTA is a better predictor of CORT parameters than the early-life conditions to which birds have been exposed; and that there are modest familial effects on baseline and peak CORT. In addition, our study, through having two age points, gave us the opportunity to ask a number of additional questions. First, it may be that the association between DTA and the strength of the stress response develops with chronological age. Our age point 2 was most similar to the ages of the birds in the study by Andrews et al. (2017). Thus, it is possible that the association between DTA and CORT variables will be observable at age point 2 , but not yet in evidence at age point 1 . Second, in their interpretation of their findings, Andrews et al. (2017) assumed that the strength of the stress response declines with chronological age in the starling, basing this assumption on published literature from other bird species. In our study, we were able to directly test this assumption within individuals. Third, our repeated-measure design allowed us to characterise individual consistency in CORT variables in the starling. 
Our study suffered from a large limitation in respect of the analyses of within-individual change with chronological age, and individual consistency. Unfortunately, the second age point sample was not planned at the time the first was carried out. We therefore performed the laboratory assays on the blood samples from the first age point separately to the second, and with a small variation in the laboratory protocol. Although common standard samples were run on both occasions, this does limit the robustness of inferences we can make about within-individual change and consistency, since age point and laboratory batch are completely confounded. The findings in respect of within-individual change with age, and individual consistency, should therefore be interpreted with caution.

\section{MATERIALS AND METHODS}

\section{Study subjects and husbandry}

Study subjects were from a cohort of 32 starlings (16 male) hatched in 2014 (hence the '2014 cohort') described in detail elsewhere (Nettle et al., 2017). Briefly, quartets of natural nest-mates were taken from the wild on day 5 post-hatch and hand-reared. One sibling was assigned to each combination of food Amount ('Plenty' vs. 'Lean') and begging Effort ('Easy'vs. 'Hard'), thus creating four experimental groups of eight birds per group. The Amount manipulation was achieved by feeding each Plenty group to satiation on each feed and measuring the quantity consumed, then restricting the intake of the corresponding Lean group to $73 \%$ of this quantity. The effort manipulation was achieved by interspersing, for the Hard groups, each true feed with another nest visit of similar duration where the nestlings were stimulated to beg but no food was delivered. The experimental treatments lasted until day 15, after which all birds were hand-fed ad libitum until independence. Birds were subsequently kept in mixed-treatment flocks in indoor aviaries $\left(215 \times 340 \times 220 \mathrm{~cm} ; 18{ }^{\circ} \mathrm{C}, 40 \%\right.$ humidity $)$, with ad libitum access to food and water. Birds were maintained in non-breeding conditions by a constant $15 \mathrm{~h}$ light: 9 h dark cycle.

Our study adhered to the ASAB/ABS Guidelines for the Use of Animals in Research, and was approved by the Animal Welfare and Ethics Review Board at Newcastle University. It was completed under UK Home Office project licence number PPL 70/8089.

\section{Developmental telomere attrition}

Telomere length was measured in erythrocyte DNA from blood samples taken on day 5 and day 56 post-hatch using a real-time PCR amplification method (for details see Nettle et al., 2017). We used the two T/S ratio values to gain a single-number summary of telomere shortening (henceforth $\Delta \mathrm{TL}$ ). $\Delta \mathrm{TL}$ was corrected for the regression to the mean using the method of Verhulst et al. (2013). Consequently, 0 represents the average amount of change for the cohort, and a negative number more dramatic shortening. Similar results are obtained using the raw difference in $\mathrm{T} / \mathrm{S}$ ratios instead. 


\section{Blood sampling and corticosterone assays}

Birds were aged 127-134 days at age point 1, and 584-601 days at age point 2. For the period of sampling, birds were individually caged $(75 \times 45 \times 45 \mathrm{~cm})$ whilst maintaining full acoustic and visual contact with others. The minimum time in individual cages prior to sampling was 3 days. All birds had access to two wooden perches, two drinking bottles, a water bath (removed approximately $4 \mathrm{~h}$ prior to sampling) and a bowl containing ad libitum food. The birds were maintained in environmental conditions identical to the free-flight aviaries. Habituation to the cages occurred over a minimum of three nights prior to blood sampling.

At a set time during the afternoon (the period of minimal diurnal CORT variation, Romero \& Remage-Healey, 2000), lights were extinguished and two birds caught from their cages and transferred immediately to an adjacent procedure room. Approximately $120 \mu \mathrm{l}$ of a baseline blood sample was collected within $3 \mathrm{~min}$ of the lights being extinguished (age 1: mean time to baseline sample: $94.9 \pm 23.6 \mathrm{~s}$; age 2: $108.4 \pm 29.7 \mathrm{~s}$ ). One bird was removed from analysis at age 1 as the time to baseline sample was at the $180 \mathrm{~s}$ limit and visual analysis of the radioimmunoassay data confirmed a very high level of baseline CORT. Bleeding was stemmed using cotton wool and birds were placed in drawstring cloth bags. Further samples were taken at 15 and 30 min after the initial disturbance. Blood was taken by puncture of an alar or metatarsal vein, and collection by heparinised micro-capillary tubes. After the final sample, birds were weighed and returned to the cage under observation. Each experimental room was disturbed for sampling only once per day, and no-one entered the room for at least $2 \mathrm{~h}$ prior to a sample being taken.

Blood samples were centrifuged (10 min at 3,000 rpm) to separate plasma from erythrocytes and stored at $-80{ }^{\circ} \mathrm{C}$ until radioimmunoassay analysis. Samples from age point 1 and age point 2 were analysed at different times. Batch 1 (age point 1 ) consisted of two separate assays, and batch 2 (age point 2) consisted of four assays. The same protocol was followed for both batches, and two standard chicken plasma samples (P3266-1ML; Sigma Aldrich, St. Louis, MO, USA) were run in all assays in both batches. All samples were run in duplicate within the same assay. Freezer storage times were approximately 120 days (batch 1 ) and 30 days (batch 2 ).

Corticosterone levels in plasma extracts were quantified using a radioimmunoassay previously validated in European starlings (Buchanan et al., 2003). CORT concentrations were measured after extraction of up to $35 \mu \mathrm{l}$ aliquots of plasma in one ml diethyl ether (24004-2.5L-M; Sigma Aldrich, St. Louis, MO, USA) by a Dextran 70-coated charcoal radioimmunoassay method. The anti-CORT serum was ABIN880 (Antibodies Online, London, UK) for batch 1 and 07120016 (MP Biomedical, Loughborough, UK) for batch 2. Extraction efficiencies per sample were estimated at 61-100\% (mean 97.8\%) for batch 1, and 77-100\% (mean 96.1\%) for batch 2. Final CORT concentration values were corrected accordingly.

The average intra-assay coefficient of variation was $8.7 \%$ for batch 1 and $13.5 \%$ for batch 2 . The inter-assay coefficient of variation (collapsing across batches) was $21.9 \%$. 
The batch 2 average concentration values for the two control samples were $99.84 \%$ and $78.15 \%$ of the batch 1 values, respectively.

\section{Data analysis}

As in Andrews et al. (2017), the dynamics of the stress response were characterised by three dependent variables: baseline CORT (the first sample value); peak CORT (higher of second and third sample values); and $\triangle$ CORT (the change in CORT value between 15 and $30 \mathrm{~min}$ samples, with a negative number indicating a reduction from 15 to $30 \mathrm{~min}$ ).

Our main data-analytic approach was to fit linear mixed models of the data from age points 1 and 2 combined, for each of the three outcome variables (baseline CORT, peak CORT, and $\triangle \mathrm{CORT}$ ), in turn. All models contained random effects of bird, nested within natal family. To examine individual consistency and familial resemblance, we fitted initial models with no fixed predictors. The proportion of variation explained by the random effect of bird in these initial models is a measure of individual consistency. An alternative approach to estimating individual consistency is to calculate the intra-class correlation coefficient between the age point 1 and age point 2 measures. We also did this, and report these in the 'Results' section. The extent of familial resemblance can be estimated from the initial models using the proportion of variation explained by the random effect of natal family.

For the main objectives, we added $\Delta \mathrm{TL}$, age point, and the interaction between $\Delta \mathrm{TL}$ and age point to the initial models as fixed predictors. These models thus test for within-individual changes with chronological age; associations between $\Delta \mathrm{TL}$ and CORT variables after controlling for age point; as well as differences between the age points in terms of the association between $\Delta \mathrm{TL}$ and the outcome variable. As in Andrews et al. (2017), we included baseline CORT as a covariate in the model where the outcome variable was peak CORT; and CORT at $15 \mathrm{~min}$ as a covariate in the model where the outcome variable was $\Delta$ CORT. We did not include additional covariates (sex, body weight, time elapsed before baseline sample) that we might otherwise have considered, since these were not included in the analyses of the previous paper. We can report that including these does not substantively alter the results presented here.

There are two drawbacks to our modelling approach: first, it does not involve exactly the same statistical models as Andrews et al. (2017), since their data included only one age point; and second, it combines the data from the two laboratory batches, which could be misleading if the assays from the two batches are not validly comparable. For the

replication of the associations with $\Delta \mathrm{TL}$, we therefore also ran additional analyses treating the age point 1 and age point 2 datasets as completely separate, and for each one in turn, fitting the same statistical models as Andrews et al. (2017).

In order to establish the current balance of evidence on associations between $\Delta \mathrm{TL}$ and CORT variables, we performed fixed-effects meta-analyses on all three cohorts of birds (the two from the previous paper plus the present one; the cohorts from the earlier paper are referred to as 2012 and 2013). Separate meta-analyses were performed using age point 1 to represent the 2014 cohort, and using age point 2. Parameter estimates were recalculated as standardised $\beta$ s for this purpose. 
To investigate effects of developmental treatments, we fitted models of each CORT variable with Amount, Effort, age point and all their two-way interactions as fixed predictors. Again, baseline CORT was included as a covariate for the model of peak CORT, and CORT at $15 \mathrm{~min}$ for the model of $\triangle$ CORT.

All analyses were performed in R Version 3.5.0 (R Core Development Team, 2018), using the contributed packages 'irr' (Gamer et al., 2012) for intra-class correlation coefficients, 'afex' (Singmann et al., 2018) for linear mixed models, and 'metafor' (Viechtbauer, 2010) for meta-analysis. Categorical variables (the developmental treatments) are automatically contrast-coded by the 'afex' package. Type-III significance tests for linear mixed models were by likelihood ratio test (LRT) with a significance threshold of 0.05 , and hence parameter estimation was by maximum likelihood.

We also created a simple $\mathrm{R}$ simulation tool to simulate the chances of finding a significant $(p<0.05)$ association between two variables in small samples, for a given true strength of association. This tool generates 10,000 samples of a specified size from datasets where the true strength of association is as specified, and tabulates how many of them find a significant effect, how many a non-significant effect but in the predicted direction, and how many an effect in the opposite direction. This tool thus simulates the power of either our age point 1 or age point 2 data considered separately to replicate the significant associations observed in Andrews et al. (2017).

Raw data and R scripts are available for download from: https://doi.org/10.5281/ zenodo.1317793.

\section{RESULTS}

\section{Final sample sizes}

Of the original 32 birds in the cohort, we failed to obtain CORT variables at age point 1 for one bird, and two birds died between age point 1 and age point 2 . Thus, maximum samples sizes for CORT variables were 31 for age point 1 and 30 for age point 2, with 29 birds having data at both time points. In addition, due to telomere assay failures (see Nettle et al., 2017), $\Delta$ TL was unavailable for five birds, including one of the birds that died between age point 1 and age point 2 . Thus, for analyses involving $\Delta T L$, the maximum sample size is 26 at age point 1 and 26 at age point 2, with 25 birds having data at both time points.

\section{Descriptive statistics}

Descriptive statistics for CORT values at all time points and both age points are shown in Table 1. At both age points, the baseline blood sample gave the lowest CORT value for all birds (Fig. 1). Some birds' CORT values were highest at $15 \mathrm{~min}$ and then declined by $30 \mathrm{~min}$, giving $\Delta$ CORT values less than 0 (16/31 at age point $1,16 / 30$ at age point 2 ). The remainder continued to show an increase between 15 and $30 \mathrm{~min}$.

\section{Individual consistency and familial effects}

Our initial models contained only the random effects of bird nested within natal family. For baseline CORT and peak CORT, bird explained $<1 \%$ of the variation in the initial models. This suggests negligible individual consistency in these CORT variables. This was 
Table 1 Descriptive statistics (mean \pm SD) for CORT variables $(\mathrm{ng} / \mathrm{ml})$ at age point 1 and age point 2 .

\begin{tabular}{lll} 
Variable & Age $\mathbf{1}$ (127-134 days) & Age 2 (584-601 days) \\
\hline Baseline CORT & $2.21 \pm 1.99$ & $2.76 \pm 1.53$ \\
CORT 15 min & $19.58 \pm 8.89$ & $13.89 \pm 6.23$ \\
CORT 30 min & $18.44 \pm 6.41$ & $13.25 \pm 5.27$ \\
Peak CORT & $21.66 \pm 8.50$ & $15.48 \pm 5.77$ \\
$\Delta$ CORT & $-1.14 \pm 7.00$ & $-0.64 \pm 5.01$ \\
\hline
\end{tabular}

Note:

Peak CORT represents the higher of CORT $15 \mathrm{~min}$ and CORT $30 \mathrm{~min} ; \Delta$ CORT represents CORT $30 \mathrm{~min}$ minus CORT $15 \mathrm{~min}$.

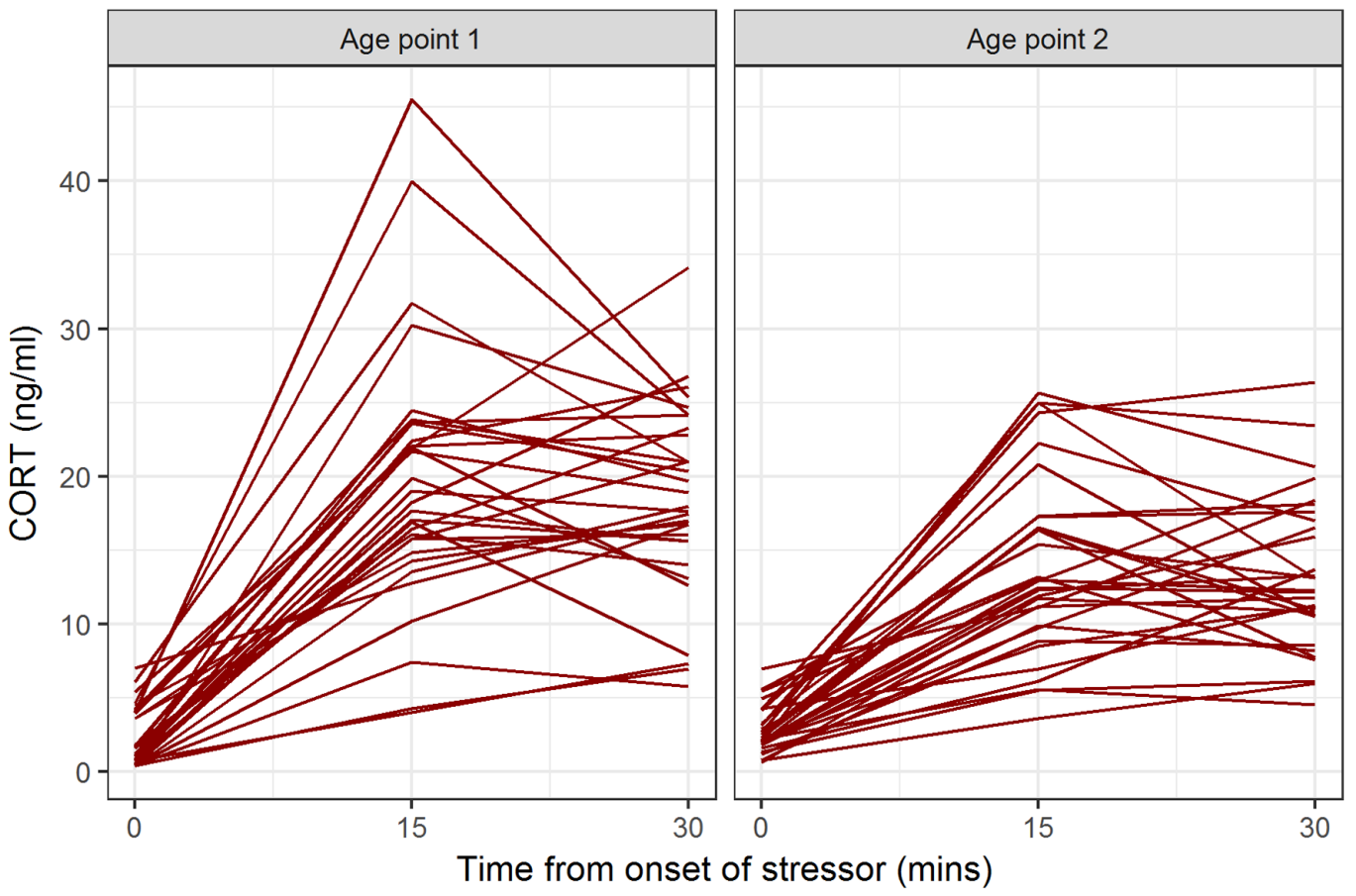

Figure 1 CORT values for individual birds at baseline, $15 \mathrm{~min}$ after onset of stressor, and $30 \mathrm{~min}$ after onset of stressor, at the two age points. Full-size $\boldsymbol{0}$ DOI: $10.7717 /$ peerj.5842/fig-1

confirmed by calculating intra-class correlation coefficients between age point 1 and age point 2 (baseline CORT $0.13,95 \%$ CI [ -0.24 to 0.46$]$; peak CORT $-0.03,95 \%$ CI [ -0.38 to $0.33]$ ). For $\Delta$ CORT, bird explained $35 \%$ of the variation, suggesting some degree of individual consistency. This was confirmed by the intra-class correlation coefficient, which was higher than for the other two CORT variables, though its $95 \%$ confidence interval still included zero $(0.32,95 \%$ CI $[-0.04$ to 0.61$])$.

Natal nest explained $33 \%$ of the variation in baseline CORT; $17 \%$ of the variation in peak CORT; and $<1 \%$ of the variation in $\Delta$ CORT (Fig. 2).

\section{Effects of chronological age and $\Delta \mathrm{TL}$}

Our main models included fixed effects of age point, $\Delta \mathrm{TL}$, and their interaction.

A complete replication of Andrews et al. (2017) would find significant main effects of 

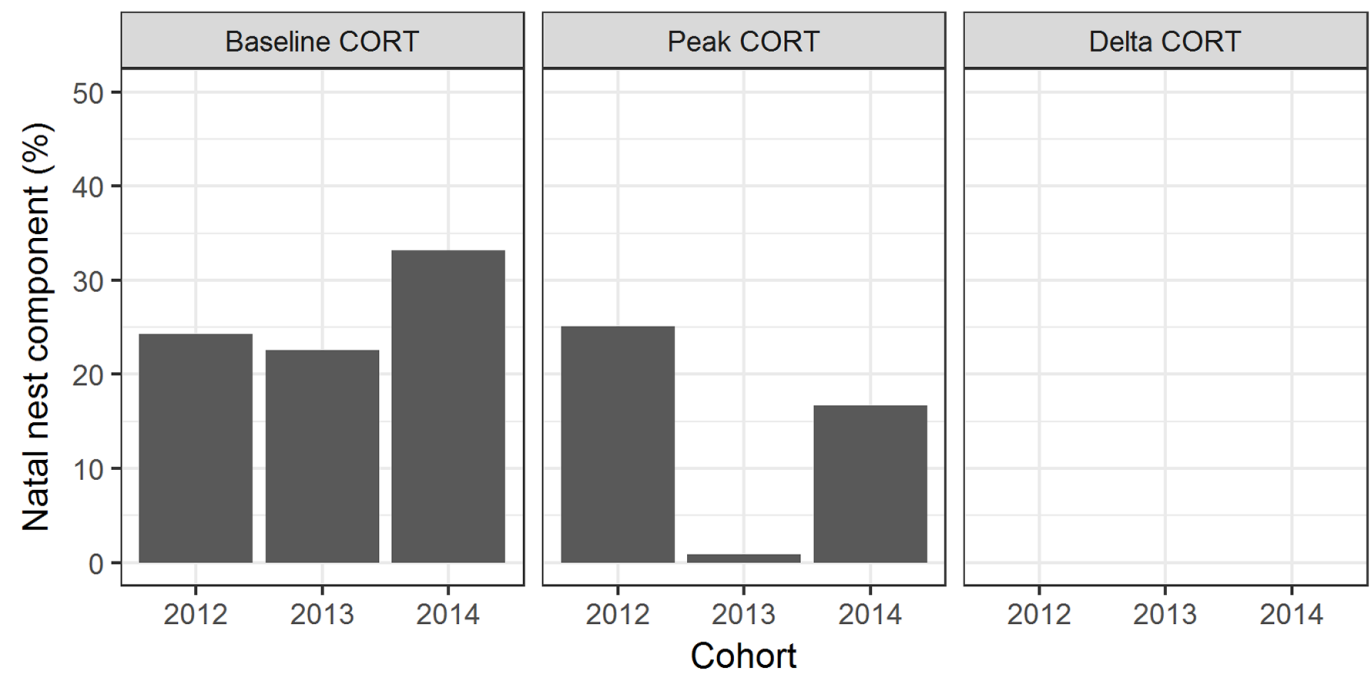

Figure 2 Familial and individual components of variation in CORT variables. Bars represent the proportion of variation explained by natal nest and bird, for the two cohorts of birds studied by Andrews et al. (2017; cohorts referred to as 2012 and 2013), and the cohort studied in the present paper (2014). Full-size $\underset{0}{0}$ DOI: 10.7717 /peerj.5842/fig-2

Table 2 Summaries of statistical models testing for effects of age point and developmental telomere attrition (DTA) on stress response (CORT) variables.

\begin{tabular}{llllll} 
Outcome variable & Fixed predictors & B & s.e. $(\mathbf{B})$ & LRT & p-value \\
\hline Baseline CORT & Age point & 0.51 & 0.40 & 1.65 & 0.20 \\
& DTA & -0.66 & 1.25 & 0.28 & 0.60 \\
\multirow{3}{*}{ Peak CORT } & Age point $\times$ DTA & 2.54 & 1.66 & 2.27 & 0.13 \\
& Baseline CORT & 0.55 & 0.55 & 0.92 & 0.34 \\
& Age point & -5.82 & 1.64 & 11.18 & $<0.001^{*}$ \\
$\Delta$ CORT & DTA & 3.02 & 5.09 & 0.35 & 0.55 \\
& Age point $\times$ DTA & -4.37 & 6.93 & 0.39 & 0.53 \\
& CORT 15 min & -0.61 & 0.08 & 33.10 & $<0.001^{*}$ \\
& Age point & -2.69 & 1.12 & 5.26 & $0.02^{*}$ \\
& DTA & -0.03 & 3.24 & 0.00 & 0.99 \\
\hline
\end{tabular}

Notes:

All models contain random effects of bird nested within family.

LRT, likelihood ratio test. " $p<0.05$.

$\Delta \mathrm{TL}$ on peak CORT and $\Delta$ CORT but not baseline CORT; a replication at one but not the other age point would find a significant interaction between age point and $\Delta \mathrm{TL}$ for peak CORT and $\triangle$ CORT but not baseline CORT; and a decline in stress response with chronological age would produce a significant main effect of age point on peak CORT and $\triangle$ CORT.

The models are summarised in Table 2. For baseline CORT, neither age point, $\Delta \mathrm{TL}$, nor their interaction were significant predictors. For peak CORT, there was a significant main effect of age point, with lower peak CORT at age point 2 
(estimated marginal means: age point 1, 21.57, 95\% CI [17.76-25.38]; age point 2, 15.74, 95\% CI [11.94-19.53]). The main effect of $\Delta \mathrm{TL}$ and the interaction between $\Delta \mathrm{TL}$ and age point were not significant. For $\Delta$ CORT, there was a significant effect of CORT at $15 \mathrm{~min}$, which was expected since birds with higher CORT at $15 \mathrm{~min}$ tend to reduce more by $30 \mathrm{~min}$. There was a significant effect of age point, with birds at age point 2 returning more towards baseline between 15 and $30 \mathrm{~min}$ (i.e. having a more negative $\Delta$ CORT value; estimated marginal means: age point $1,0.27,95 \%$ CI [-2.37 to 2.90$]$; age point $2,-2.41,-5.01$ to 0.19 ). The main effect of $\Delta \mathrm{TL}$ and its interaction with age point were not significant.

We additionally performed analyses on the two age point datasets separately, fitting to each one exactly the same statistical models used in the study by Andrews et al. (2017). These models are summarised in Table S1; they lead to the same conclusion that $\Delta \mathrm{TL}$ does not significantly predict any CORT variable in the present cohort of birds.

\section{Meta-analysis}

The lack of significant effects involving $\Delta \mathrm{TL}$ in the present study contrasts with the findings of Andrews et al. (2017). To evaluate the balance of evidence, we performed fixed-effects meta-analyses combining the data from the present study with the data from the earlier paper (Fig. 3). As the figure shows, the summary association between $\Delta \mathrm{TL}$ and baseline CORT was not significantly different from zero, regardless of whether the age point 1 or age point 2 results were used to represent the 2014 cohort. The association between $\Delta \mathrm{TL}$ and peak CORT after controlling for baseline CORT was significantly positive (that is, more telomere loss associated with a lower peak CORT concentration) if the age point 1 data were used to represent the 2014 cohort ( $\beta=0.30,95 \%$ CI $[0.01-0.41], p=0.04)$, but not significantly different from zero if the age point 2 data were used ( $\beta=0.16,95 \%$ CI $[-0.04$ to 0.36$], p=0.12$ ). The summary association between $\Delta \mathrm{TL}$ and $\Delta \mathrm{CORT}$ after controlling for CORT at $15 \mathrm{~min}$ was significantly positive (that is, more telomere loss, more CORT return towards baseline between 15 and $30 \mathrm{~min}$ after onset of stressor). This was true using either the age point $1(\beta=0.30,95 \%$ CI $[0.11-0.49], p<0.01)$ or age point 2 data $(\beta=0.30$, 95\% CI [0.11-0.49], $p<0.01)$.

\section{Effects of developmental treatment}

Table 3 summarises the models using the two developmental treatments (Amount and Effort), plus age point and their interactions, as the predictors. There were no significant main effects or interactions involving the developmental treatments for baseline CORT or peak CORT. For $\Delta$ CORT, there was a significant interaction between age point and the Effort treatment. Birds from the Hard groups showed more return of CORT towards baseline between 15 and $30 \mathrm{~min}$ (i.e. had more negative values of $\Delta \mathrm{CORT}$ ) than birds from the Easy groups at age point 1 . However, at age point 2, the $\Delta$ CORT of the birds from the Hard groups had scarcely changed on average, whereas the Easy groups had showed substantially reduced $\Delta$ CORT with age (Fig. 4). 


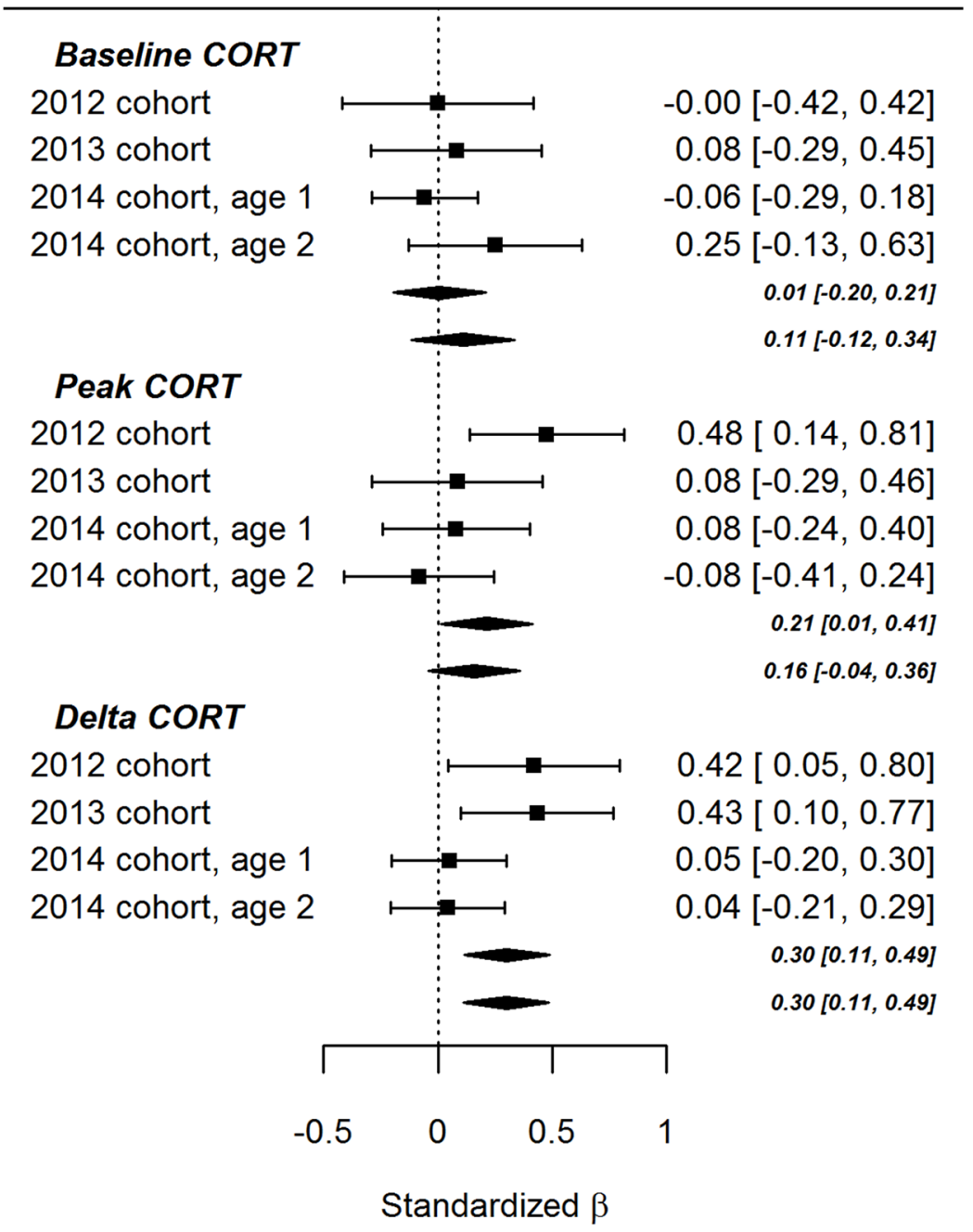

Figure 3 Forest plot of associations between developmental telomere change and CORT variables in the present cohort of birds (2014) and the two cohorts described previously (2012 and 2013). The points and whiskers show standardised parameter estimates and their $95 \%$ confidence intervals. The lozenges show summary effects from meta-analytically combining the three datasets (upper lozenges use the age 1 point data for the present cohort, lower lozenges use the age point 2 data).

Full-size $\underline{0}$ DOI: 10.7717 /peerj.5842/fig-3

\section{Power simulation}

To set our failure to replicate the significant patterns involving $\Delta$ TL reported in the previous paper into context, we simulated 10,000 samples of 27 individuals from populations where the 'true' association between $\Delta \mathrm{TL}$ and peak CORT was 0.28 , which is the pooled estimate from the two cohorts of birds reported in Andrews et al. (2017). Of these 10,000 samples, $30 \%$ produced 'significant' estimates of association with $p<0.05 ; 63 \%$ produced non-significant associations but with an estimate in the positive direction; and $7 \%$ produced estimates of association in the other direction. We repeated the same exercise for the estimate of association between $\Delta \mathrm{TL}$ and $\Delta \mathrm{CORT}(0.43)$. This produced 'significant' associations $64 \%$ of the time, non-significant associations in the same direction 35\% of the time, and estimated associations in the opposite direction $1 \%$ of the time. 
Table 3 Summaries of statistical models testing for effects of developmental treatments on stress response (CORT) variables.

\begin{tabular}{|c|c|c|c|c|c|}
\hline Outcome & Fixed predictors & B & s.e. (B) & LRT & $p$-value \\
\hline \multirow[t]{6}{*}{ Baseline CORT } & Age point & 0.48 & 0.38 & 1.61 & 0.20 \\
\hline & Amount & 0.03 & 0.26 & 0.02 & 0.90 \\
\hline & Effort & -0.06 & 0.26 & 0.05 & 0.81 \\
\hline & Amount $\times$ Effort & 0.11 & 0.19 & 0.33 & 0.57 \\
\hline & Amount $\times$ Age point & -0.13 & 0.37 & 0.11 & 0.74 \\
\hline & Effort $\times$ Age point & -0.02 & 0.38 & 0.00 & 0.97 \\
\hline \multirow[t]{7}{*}{ Peak CORT } & Baseline CORT & 1.06 & 0.51 & 4.00 & $0.05^{*}$ \\
\hline & Age point & -6.69 & 1.59 & 15.43 & $<0.001^{*}$ \\
\hline & Amount & 1.40 & 1.10 & 1.62 & 0.20 \\
\hline & Effort & 1.17 & 1.10 & 1.13 & 0.29 \\
\hline & Amount $\times$ Effort & -0.41 & 0.78 & 0.27 & 0.60 \\
\hline & Amount $\times$ Age point & -1.34 & 1.57 & 0.73 & 0.39 \\
\hline & Effort $\times$ Age point & -2.18 & 1.57 & 1.90 & 0.17 \\
\hline \multirow[t]{7}{*}{$\Delta \mathrm{CORT}$} & CORT $15 \mathrm{~min}$ & -0.58 & 0.07 & 38.71 & $<0.001^{*}$ \\
\hline & Age point & -2.77 & 1.03 & 6.58 & $0.01^{*}$ \\
\hline & Amount & -0.53 & 0.69 & 0.59 & 0.44 \\
\hline & Effort & 1.37 & 0.67 & 3.99 & $0.05^{*}$ \\
\hline & Amount $\times$ Effort & 0.24 & 0.48 & 0.24 & 0.62 \\
\hline & Amount $\times$ Age point & 0.50 & 0.96 & 0.26 & 0.61 \\
\hline & Effort $\times$ Age point & -2.55 & 0.95 & 6.68 & $0.01^{*}$ \\
\hline
\end{tabular}

Notes:

All models contain random effects of bird nested within natal family.

LRT, likelihood ratio test.

${ }^{*} p<0.05$.

\section{DISCUSSION}

The main aim of our experiment was to replicate the findings of Andrews et al.'s (2017) study in a different cohort of birds measured at two time points. We did not replicate the main patterns observed in the previous paper concerning DTA. The change in erythrocyte telomere length over development did not significantly predict any of the CORT parameters in the present cohort, in the whole dataset, or at either age point considered separately. Our failure to clearly replicate the patterns of the previous study does not support the conclusions of that paper. However, we should not necessarily infer that those conclusions were spurious, either. Meta-analysis of all the extant evidence supports a moderate association between DTA and $\triangle$ CORT $(\beta=0.30)$, with starlings that have experienced more DTA showing more rapid recovery of CORT towards baseline; and possibly between DTA and peak CORT, with starlings that have experienced greater DTA having a lower peak. The former conclusion is supported whether age point 1 or age point 2 is used to represent the present 2014 cohort of birds. The latter conclusion is only supported if the age point 1 data are used. Age point 2 (584-601 days) was more similar to the age at which the birds' stress responses were measured in the previous study (2012 cohort, 208-432 days; 2013 cohort, 428-456 days). Our simulations 

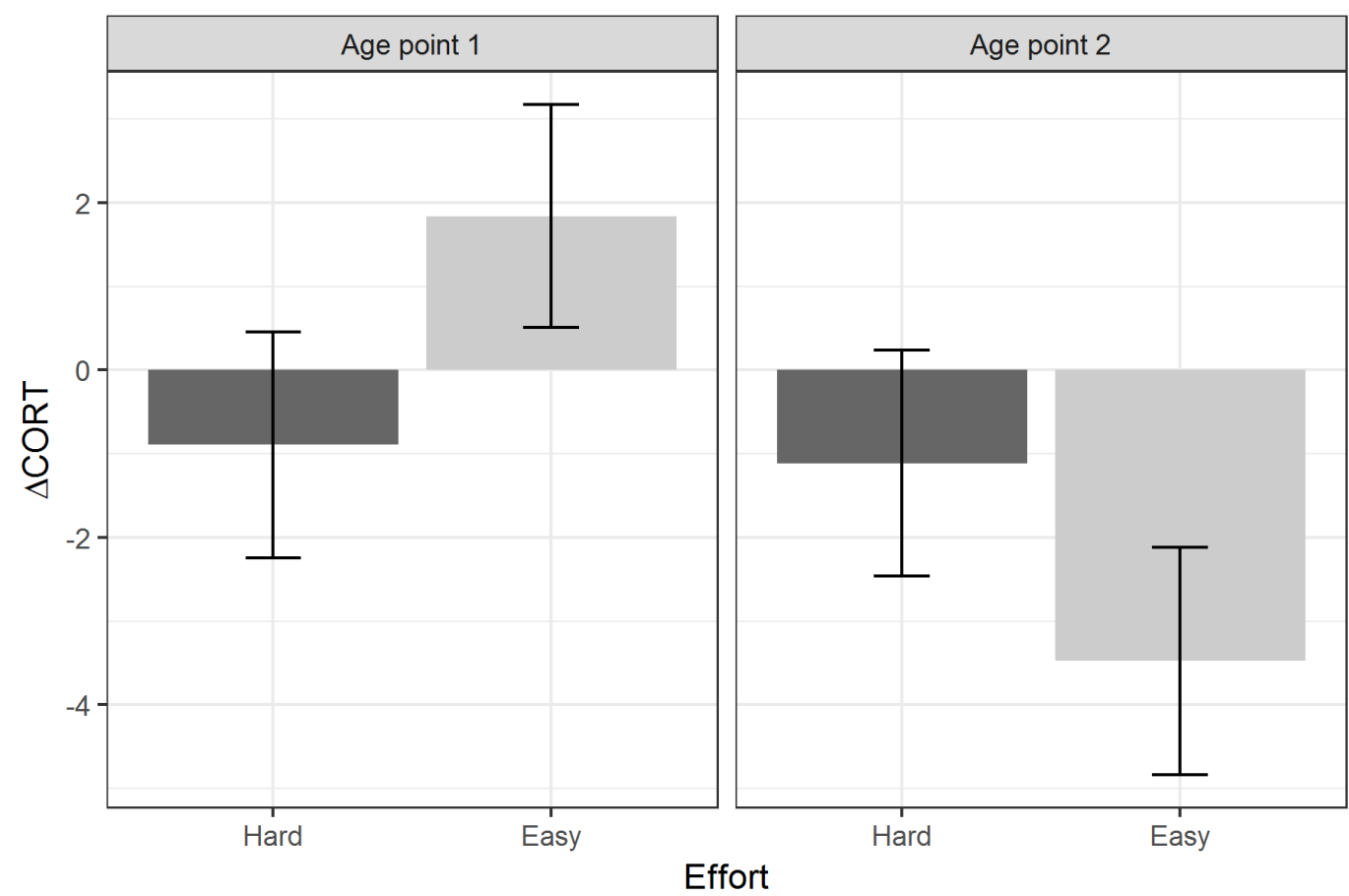

Figure 4 Estimated marginal means of $\Delta$ CORT after controlling for CORT at $15 \mathrm{~min}$, by levels of the Effort developmental treatment, and age point. Error bars represent plus/minus one standard error. Full-size

showed that if associations between DTA and CORT variables do in fact exist, and have the strength estimated in Andrews et al. (2017), then we should not expect them to be statistically 'significant' (i.e. have $p<0.05$ ) in every small- $n$ sample considered individually. For example, if the estimated parameters in Andrews et al. (2017) are correct, then we should only expect a 'significant' finding about one experiment in three for peak CORT, and two in three for $\triangle \mathrm{CORT}$. (Our two age points constitute two separate experiments in the context of this statement).

We acknowledge the low power of our experiment for detecting associations between DTA and CORT parameters. However, there are strong logistical constraints involved in capturing, keeping, in our case hand-rearing, and sampling live wild animals.

For example, to detect an association of $\beta=0.30$ with the conventional $80 \%$ power requires over 80 birds, which is beyond the population size of our starling breeding colony or our ability to hand-rear nestlings under precisely controlled conditions. This means that modest sample sizes are difficult to avoid, especially in the early stages of exploration of certain questions. Given that we cannot rear very large cohorts of birds, we have to turn to sequential replication and cumulative meta-analysis, rather than individual-experiment $p$-values, as a way of ensuring robustness of conclusions.

A further limitation of both this study and Andrews et al. (2017) is that 'biological age' is proxied by a single marker, DTA. A better approach for capturing the biological age construct is to use a whole panel of biomarkers, each of which has been shown to predict subsequent lifespan (Levine, 2013; Belsky et al., 2015). Such a panel predicts morbidity 
and other phenotypic outcomes better than any single constituent biomarker, each of which might have quite weak predictive power. Thus, a stronger test of the hypothesis that individual differences in stress response among birds of the same chronological age are explained by differences in their biological ages would use a panel of multiple biomarkers of biological age, rather than the single one used here.

The three CORT parameters were not individually consistent across the two age points (only $\triangle$ CORT showed any suggestion of individual consistency across the two age points, and this was still fairly low). This must be interpreted cautiously given that the samples from the two age points were run in separate laboratory batches. It does however concur with some previous findings in the avian literature that individual consistency can be low (Ouyang, Hau \& Bonier, 2011; Baugh et al., 2014; Lendvai, Giraudeau \& Bo, 2015); and if consistency is found, is in the response to acute stress rather than baseline CORT (Cockrem \& Silverin, 2002; Rensel \& Schoech, 2011).

We found some evidence of familial effects. Specifically, natal nest explained a modest but non-zero proportion of the variation in baseline CORT and peak CORT, but a negligible amount of the variation in $\triangle \mathrm{CORT}$. The familial effect findings were extremely similar to those of Andrews et al. (2017). Since our birds were removed from the wild on day 5 post-hatching, and siblings were thereafter assigned to different developmental treatment groups, these familial effects must represent either genetics, maternal effects, or very early developmental influences.

We found significant effects of chronological age on both peak CORT (lower at age point 2) and $\triangle$ CORT (more return towards baseline at age point 2). Our design was more powerful for detecting these than it was for detecting associations with DTA, as the critical comparisons were within subjects. The findings should be interpreted cautiously, as chronological age was completely confounded with laboratory batch. Nonetheless, they may at least partly represent within-individual biological change with age. There were common control samples run in both batches; the average concentration for these standards at age point 2 was $99.84 \%$ and $78.15 \%$ of their age point 1 values, whereas peak CORT was $71.47 \%$ of its average age 1 value. Decline in the strength of the CORT response (but not baseline CORT) with chronological age is a very widely observed pattern in birds (Heidinger, Nisbet \& Ketterson, 2006; Heidinger et al., 2010; Wilcoxen et al., 2011; Elliott et al., 2014; Lendvai, Giraudeau \& Bo, 2015; López-Jiménez et al., 2017). Indeed, a chronological age-related decline in CORT response is one of the key assumptions on which Andrews et al. (2017) based their hypotheses, but which they did not directly test. Thus, we have provided some validating evidence for this part of their argument. The differences between age point 1 and age point 2 were strikingly large, and set the associations between DTA and CORT parameters in context. They suggest that any DTA-related differences in stress response amongst birds of the same age are small relative to the within-bird change as a bird develops from a juvenile into an adult.

We found some evidence that one of the developmental manipulations to which the birds were subjected may have affected their stress responses, in an age-dependent manner. Specifically, age point and begging Effort interacted to predict $\Delta$ CORT. 
At the first age point, birds from the Hard begging effort groups had more negative values of $\Delta$ CORT than those from the Easy groups. This is consistent with the idea that increased begging effort accelerates biological ageing, since $\Delta$ CORT values become more negative with chronological age. It is also consilient with the finding that Hard begging effort accelerated telomere shortening in early life in these birds (Nettle et al., 2017). However, at the second age point, the average $\Delta$ CORT had greatly reduced for the Easy birds, who showed the general age-related trend, whilst for the Hard birds it had remained almost unchanged compared to age point 1 . Thus, the pattern cannot simply be described as begging effort increasing the rate of ageing of the stress response. Rather, increased begging effort produces in a juvenile bird the stress response that would be expected of an adult; and then that stress response remains the same into adulthood, whilst the stress responses of other birds are changing substantially. Thus, the shape of age-related pattern appears to be different for the Hard birds than for the Easy birds. We note that these developmental treatment findings were not predicted a priori; thus, we view these treatment-related findings as exploratory. However, other studies of these same birds have also found that the Hard Effort treatment leaves a lasting phenotypic legacy: Dunn et al. (2018) showed that the Hard birds maintain a lower body mass than the Easy birds through adulthood, and correspondingly employ different foraging strategies.

In Andrews et al. (2017), a major finding was that developmental conditions did not significantly predict stress parameters, whereas DTA did, the converse of what we see here. Given that developmental treatments were significantly related to DTA in all the cohorts of birds that we have studied, the fact that significant predictor of the strength of the stress response was sometimes DTA (Andrews et al., 2017), and sometimes the developmental treatment itself (here), may relate to limited statistical power, as discussed above.

\section{CONCLUSIONS}

In conclusion, we did not confirm any significant associations between biological age, as proxied by DTA, and the strength of the stress response, as measured by CORT response to an acute capture-restraint-handling stressor, in a cohort of hand-reared European starlings. However, the overall evidence is still consistent with biological age being associated with aspects of the stress response in starlings. Moreover, our data suggest there is a substantial decline in the strength of the stress response with chronological age in the starling, as the biological age hypothesis requires. They also suggest that early-life begging effort may affect the strength of the stress response in an age-dependent manner; and confirm earlier observations of familial effects on baseline and peak CORT.

\section{ACKNOWLEDGEMENTS}

We thank Pat Monaghan and Sophie Reichert, who collaborated with us on the telomere attrition of the birds studied in this paper; and Tom Bedford and Michelle Waddle, who assisted with us in the care of the birds. 


\section{ADDITIONAL INFORMATION AND DECLARATIONS}

\section{Funding}

This research was funded by the Biotechnology and Biological Sciences Research Council (BBSRC) under grants BB/J016446/1 and BB/J016292/1; a doctoral training studentship to Annie Gott; and a David Phillips fellowship to Karen Spencer. The project has also received funding from the European Research Council (ERC) under the European Union's Horizon 2020 research and innovation programme (grant agreement no. AdG 666669 (COMSTAR)). The funders had no role in study design, data collection and analysis, decision to publish, or preparation of the manuscript.

\section{Grant Disclosures}

The following grant information was disclosed by the authors:

Biotechnology and Biological Sciences Research Council (BBSRC) under grants: BB/J016446/1 and BB/J016292/1.

European Research Council (ERC) under the European Union's Horizon 2020 research and innovation programme: AdG 666669 (COMSTAR).

\section{Competing Interests}

The authors declare that they have no competing interests.

\section{Author Contributions}

- Annie Gott conceived and designed the experiments, performed the experiments, analysed the data, prepared figures and/or tables, authored or reviewed drafts of the paper, approved the final draft.

- Clare Andrews conceived and designed the experiments, performed the experiments, analysed the data, prepared figures and/or tables, authored or reviewed drafts of the paper, approved the final draft.

- Maria Larriva Hormigos conceived and designed the experiments, authored or reviewed drafts of the paper, approved the final draft, hormonal analysis.

- Karen Spencer conceived and designed the experiments, analysed the data, contributed reagents/materials/analysis tools, authored or reviewed drafts of the paper, approved the final draft, hormonal analysis.

- Melissa Bateson conceived and designed the experiments, performed the experiments, contributed reagents/materials/analysis tools, authored or reviewed drafts of the paper, approved the final draft.

- Daniel Nettle conceived and designed the experiments, performed the experiments, analysed the data, prepared figures and/or tables, authored or reviewed drafts of the paper, approved the final draft.

\section{Animal Ethics}

The following information was supplied relating to ethical approvals (i.e. approving body and any reference numbers):

Our study adhered to the ASAB/ABS Guidelines for the Use of Animals in Research, and was approved by the Animal Welfare and Ethics Review Board at 
Newcastle University. It was completed under UK Home Office project license number PPL 70/8089.

\section{Data Availability}

The following information was supplied regarding data availability:

Gott, Annie, Andrews, Clare, Larriva, Maria, Spencer, Karen, Bateson, Melissa, \& Nettle, Daniel. (2018). Data archive for Gott et al. 'Chronological age, biological age, and individual variation in the stress response in the European starling: A follow-up study' [Data set]. Zenodo. http://doi.org/10.5281/zenodo.1408580.

\section{Supplemental Information}

Supplemental information for this article can be found online at http://dx.doi.org/10.7717/ peerj.5842\#supplemental-information.

\section{REFERENCES}

Andrews C, Nettle D, Larriva M, Gillespie R, Reichert S, Brilot BO, Bedford T, Monaghan P, Spencer K, Bateson M. 2017. A marker of biological age explains individual variation in the strength of the adult stress response. Royal Society Open Science 4(9):171208

DOI 10.1098/rsos.171208.

Angelier F, Wingfield JC, Weimerskirch H, Chastel O. 2010. Hormonal correlates of individual quality in a long-lived bird: a test of the 'corticosterone-fitness hypothesis'. Biology Letters 6(6):846-849 DOI 10.1098/rsbl.2010.0376.

Barrett ELB, Burke TA, Hammers M, Komdeur J, Richardson DS. 2013. Telomere length and dynamics predict mortality in a wild longitudinal study. Molecular Ecology 22(1):249-259 DOI 10.1111/mec.12110.

Bauer CM, Graham JL, Abolins-Abols M, Heidinger BJ, Ketterson ED, Greives TJ. 2018. Chronological and biological age predict seasonal reproductive timing: an investigation of clutch initiation and telomeres in birds of known age. American Naturalist 191(6):777-782 DOI 10.1086/697224.

Baugh AT, Van Oers K, Dingemanse NJ, Hau M. 2014. Baseline and stress-induced glucocorticoid concentrations are not repeatable but covary within individual great tits (Parus major). General and Comparative Endocrinology 208:154-163 DOI 10.1016/j.ygcen.2014.08.014.

Belsky DW, Caspi A, Houts R, Cohen HJ, Corcoran DL, Danese A, Harrington H, Israel S, Levine ME, Schaefer JD, Sugden K, Williams B, Yashin AI, Poulton R, Moffitt TE. 2015. Quantification of biological aging in young adults. Proceedings of the National Academy of Sciences of the United States of America 112(30):E4104-E4110 DOI 10.1073/pnas.1506264112.

Bize P, Criscuolo F, Metcalfe NB, Nasir L, Monaghan P. 2009. Telomere dynamics rather than age predict life expectancy in the wild. Proceedings of the Royal Society B: Biological Sciences 276(1662):1679-1683 DOI 10.1098/rspb.2008.1817.

Boonekamp JJ, Mulder GA, Salomons HM, Dijkstra C, Verhulst S. 2014. Nestling telomere shortening, but not telomere length, reflects developmental stress and predicts survival in wild birds. Proceedings of the Royal Society B: Biological Sciences 281(1785):20133287 DOI 10.1098/rspb.2013.3287.

Boonekamp JJ, Simons MJP, Hemerik L, Verhulst S. 2013. Telomere length behaves as biomarker of somatic redundancy rather than biological age. Aging Cell 12(2):330-332 DOI 10.1111/acel.12050. 
Buchanan KL, Spencer KA, Goldsmith AR, Catchpole CK. 2003. Song as an honest signal of past developmental stress in the European starling (Sturnus vulgaris). Proceedings of the Royal Society B: Biological Sciences 270(1520):1149-1156 DOI 10.1098/rspb.2003.2330.

Cockrem JF, Silverin B. 2002. Variation within and between birds in corticosterone responses of great tits (Parus major). General and Comparative Endocrinology 125(2):197-206 DOI 10.1006/gcen.2001.7750.

Dunn JC, Andrews CP, Nettle D, Bateson M. 2018. Early-life begging effort reduces adult body mass but strengthens behavioural defence of the rate of energy intake in European starlings. Royal Society Open Science 5(5):171918 DOI 10.1098/rsos.171918.

Elliott KH, O'Reilly KM, Hatch SA, Gaston AJ, Hare JF, Anderson WG. 2014. The prudent parent meets old age: a high stress response in very old seabirds supports the terminal restraint hypothesis. Hormones and Behavior 66(5):828-837 DOI 10.1016/j.yhbeh.2014.11.001.

Fidler F, En Chee Y, Wintle BC, Burgman MA, McCarthy MA, Gordon A. 2017. Metaresearch for evaluating reproducibility in ecology and evolution. BioScience 67(3):282-289 DOI 10.1093/biosci/biw159.

Gamer M, Lemon J, Fellows I, Singh P. 2012. irr: Various coefficients of interrater reliability and agreement. $R$ package. Available at https://CRAN.R-project.org/package=irr.

Heidinger BJ, Blount JD, Boner W, Griffiths K, Metcalfe NB, Monaghan P. 2012. Telomere length in early life predicts lifespan. Proceedings of the National Academy of Sciences of the United States of America 109(5):1743-1748 DOI 10.1073/pnas.1113306109.

Heidinger BJ, Chastel O, Nisbet ICT, Ketterson ED. 2010. Mellowing with age: older parents are less responsive to a stressor in a long-lived seabird. Functional Ecology 24(5):1037-1044 DOI 10.1111/j.1365-2435.2010.01733.x.

Heidinger BJ, Nisbet ICT, Ketterson ED. 2006. Older parents are less responsive to a stressor in a long-lived seabird: a mechanism for increased reproductive performance with age? Proceedings of the Royal Society B: Biological Sciences 273(1598):2227-2231 DOI 10.1098/rspb.2006.3557.

Lendvai Z, Giraudeau M, Bo V. 2015. Within-individual plasticity explains age-related decrease in stress response in a short-lived bird. Biology Letters 11(7):20150272 DOI 10.1098/rsbl.2015.0272.

Levine ME. 2013. Modeling the rate of senescence: Can estimated biological age predict mortality more accurately than chronological age? Journals of Gerontology Series A: Biological Sciences and Medical Sciences 68(6):667-674 DOI 10.1093/gerona/gls233.

López-Jiménez L, Blas J, Tanferna A, Cabezas S, Marchant T, Hiraldo F, Sergio F. 2017. Lifetime variation in feather corticosterone levels in a long-lived raptor. Oecologia 183(2):315-326 DOI 10.1007/s00442-016-3708-0.

Nettle D, Andrews C, Reichert S, Bed T, Kolenda C, Parker C, Martin-ruiz C, Monaghan P, Bateson M. 2017. Early-life adversity accelerates cellular ageing and affects adult inflammation: experimental evidence from the European starling. Scientific Reports 7(1):40794 DOI 10.1038/srep40794.

Nettle D, Monaghan P, Boner W, Gillespie R, Bateson M. 2013. Bottom of the heap: having heavier competitors accelerates early-life telomere loss in the European starling, Sturnus vulgaris. PLOS ONE 8(12):e83617 DOI 10.1371/journal.pone.0083617.

Nettle D, Monaghan P, Gillespie R, Bedford T, Brilot B, Bateson M. 2015. An experimental demonstration that early-life competitive disadvantage accelerates telomere loss.

Proceedings of the Royal Society B: Biological Sciences 282(1798):20141610

DOI 10.1098/rspb.2014.1610. 
Ouyang JQ, Hau M, Bonier F. 2011. Within seasons and among years: when are corticosterone levels repeatable? Hormones and Behavior 60(5):559-564 DOI 10.1016/j.yhbeh.2011.08.004.

R Core Development Team. 2018. R: A Language and Environment for Statistical Computing. Vienna: The R Foundation for Statistical Computing. Available at http://www.R-project.org/.

Rensel MA, Schoech SJ. 2011. Repeatability of baseline and stress-induced corticosterone levels across early life stages in the Florida scrub-jay (Aphelocoma coerulescens).

Hormones and Behavior 59(4):497-502 DOI 10.1016/j.yhbeh.2011.01.010.

Romero LM, Remage-Healey L. 2000. Daily and seasonal variation in response to stress in captive starlings (Sturnus vulgaris): Corticosterone. General and Comparative Endocrinology 119(1):52-59 DOI 10.1006/gcen.2000.7491.

Salmón P, Nilsson JF, Watson H, Bensch S, Isaksson C. 2017. Selective disappearance of great tits with short telomeres in urban areas. Proceedings of the Royal Society B: Biological Sciences 284(1862):20171349 DOI 10.1098/rspb.2017.1349.

Singmann H, Bolker B, Westfall J, Aust F. 2018. Analysis of Factorial Experiments. R package. Available at https://CRAN.R-project.org/package $=$ afex.

Verhulst S, Aviv A, Benetos A, Berenson GS, Kark JD. 2013. Do leukocyte telomere length dynamics depend on baseline telomere length? An analysis that corrects for 'regression to the mean'. European Journal of Epidemiology 28(11):859-866 DOI 10.1007/s10654-013-9845-4.

Viechtbauer W. 2010. Conducting meta-analyses in $R$ with the metafor package. Journal of Statistical Software 36(3):1-48 DOI 10.18637/jss.v036.i03.

Wilbourn RV, Moatt JP, Froy H, Walling CA, Nussey DH, Boonekamp JJ. 2018. The relationship between telomere length and mortality risk in non-model vertebrate systems: a meta-analysis. Philosophical Transactions of the Royal Society B: Biological Sciences 373(1741):20160447 DOI 10.1098/rstb.2016.0447.

Wilcoxen TE, Boughton RK, Bridge ES, Rensel MA, Schoech SJ. 2011. Age-related differences in baseline and stress-induced corticosterone in Florida scrub-jays. General and Comparative Endocrinology 173(3):461-466 DOI 10.1016/j.ygcen.2011.07.007. 